\title{
ON THE UNFOLDING OF FOLDED SYMPLECTIC STRUCTURES
}

\author{
Ana Cannas da Silva, Victor Guillemin, and Christopher \\ WOODWARD
}

\begin{abstract}
A folded symplectic structure is a closed 2-form which is nondegenerate except on a hypersurface, and whose restriction to that hypersurface has maximal rank. We show how a compact manifold equipped with a folded symplectic structure can sometimes be broken apart, or "unfolded", into honest compact symplectic orbifolds.

A folded symplectic structure induces a spin-c structure which is canonical (up to homotopy). We describe how the index of the spin-c Dirac operator behaves with respect to unfolding.
\end{abstract}

\section{Introduction}

Two manifolds can be summed along diffeomorphic submanifolds, provided that we are given an orientation-reversing isomorphism of the normal bundles of the submanifolds. When the submanifolds are single points, the sum is the usual connected sum.

The sum operation is more subtle in a symplectic setting. Let $\left(M_{1}, \omega_{1}\right)$ and $\left(M_{2}, \omega_{2}\right)$ be compact symplectic manifolds of dimension $2 d$ and with orientations induced by the symplectic forms. In [1] Audin showed that, with some rare exceptions, there is no way of equipping the connected sum $M_{1} \# M_{2}$ with a symplectic structure that is compatible with $\omega_{1}$ and $\omega_{2}$. (She showed even more: there is no almost complex structure on $M_{1} \# M_{2}$ which is compatible with $\omega_{1}$ and $\omega_{2}$.) On the other hand, connected sums of the form $M=M_{1} \# \overline{M_{2}}$, where $\overline{M_{2}}$ has the opposite of the symplectic orientation, can sometimes be performed symplectically; for instance, in dimension $4, M_{1} \# \overline{\mathbb{C P}^{2}}$ is the (complex) blow-up of $M_{1}$ at a point.

Grossberg and Karshon proved that $M=M_{1} \# \overline{M_{2}}$ can be equipped with a spin-c structure that is compatible with $\omega_{1}$ in the usual sense and with $\omega_{2}$ under the negative orientation (see [6]). One of the goals of this paper is to show that the Grossberg-Karshon result is related to the existence of a certain type of presymplectic structure on $M$ which is compatible with the $\omega_{i}$ 's.

A folded symplectic form ${ }^{1}$ on a compact $2 d$-dimensional manifold, $M$, is a closed two-form, $\omega$, which is symplectic except along a hypersurface, $Z$, (called

Received October 27, 1998. Revised August 12, 1999.

${ }^{1}$ Many "folded" symplectic forms arise as pull-backs of symplectic forms under maps with only folding singularities $[4,15]$. 
the folding hypersurface) and, for $p \in Z$, is equal to the two-form

$$
x_{1} d x_{1} \wedge d y_{1}+d x_{2} \wedge d y_{2}+\cdots+d x_{d} \wedge d y_{d},
$$

in an appropriate Darboux coordinate system. (Thus, in particular, $Z$ is defined in this coordinate system by the equation $x_{1}=0$.) The basic facts about folded symplectic forms (most of which are well-known) are collected in $\S 2$; and in $\S 3$ we will give some examples of these forms, the simplest of which exist on the evendimensional spheres; the question of which manifolds admit folded symplectic structures will be tackled in future work.

In $\S 5$ we will prove that if $M$ is oriented and the cohomology class $[\omega]$ is the image in $H^{2}(M ; \mathbb{R})$ of an integer cohomology class, there is a natural way of associating with $\omega$ a spin-c structure. More explicitly, in $\S 4$ we will prove the following: Let $M^{+}$(respectively, $M^{-}$) be the set of points where $\omega^{d}$ agrees (resp., disagrees) with the orientation of $M$. Let $J_{0}$ be a complex structure on the tangent bundle $T(M \backslash Z)$ (i.e., an almost complex structure on $M \backslash Z$ ) which is compatible with $\omega$. Let $\mathcal{U}$ be a neighborhood of $Z$. Then the complex structure

$$
\begin{cases}J_{0} \oplus\left(\text { rotation by } 90^{\circ}\right) & \text { on } M^{+} \backslash \mathcal{U} \\ J_{0} \oplus\left(\text { rotation by }-90^{\circ}\right) & \text { on } M^{-} \backslash \mathcal{U}\end{cases}
$$

on the vector bundle

$$
T(M \backslash \mathcal{U}) \oplus \mathbb{R}^{2},
$$

extends over $M$. In other words, there exists a complex structure, $J$, on the real vector bundle $T M \oplus \mathbb{R}^{2}$, whose restriction to $M \backslash \mathcal{U}$ is the complex structure (1.2). Of course, the complex structures in $\mathbb{R}^{2}$ induced by rotations by $+90^{\circ}$ or $-90^{\circ}$ are isomorphic, hence indistinguishable. By using these signs in formula (1.2) we mean to stress that the restriction of $J$ to the trivial $\mathbb{R}^{2}$-bundle over $M$ cannot induce a complex structure on this bundle since it matches opposite orientations over the subsets $M^{+} \backslash \mathcal{U}$ and $M^{-} \backslash \mathcal{U}$.

It is not hard to see that $\omega$ and $J$ give rise in a natural way to a spin-c structure on $M$ (see $\S 5$ for details) and in $\S 6$ we will show that the Grossberg-Karshon result can be regarded as a consequence of this fact by proving that a connected sum, $M_{1} \# \overline{M_{2}}$, of two symplectic manifolds possesses a folded symplectic structure.

The folding hypersurface, $Z$, in the definition above is equipped with a canonical null-foliation whose leaves are one-dimensional. In $\S 7$ we will prove the result below (which is one of the two main results of this paper):

If the null-foliation on $Z$ is fibrating, then the folded symplectic structure on $M$ can be "unfolded".

By this we mean the following: Let $W_{i}^{0}, i=1, \ldots, N$, be the connected components of $M \backslash Z$ and let $W_{i}$ be the closure of $W_{i}^{0}$. From $W_{i}$ one gets a topological space, $M_{i}$, by identifying points, $p_{1}$ and $p_{2}$, on the boundary of $W_{i}$ if $p_{1}$ and $p_{2}$ are on the same leaf of the null-foliation. We will prove that $M_{i}$ is a compact manifold and that the folded symplectic structure on $M$ induces 
a symplectic structure (of the usual kind) on $M_{i}$. The "unfolding" of $M$ is, by definition, the operation $M \rightsquigarrow \sqcup M_{i}$. For example, let $M$ be the connected sum of compact connected $2 d$-dimensional symplectic manifolds,

$$
M=M_{1} \# \overline{M_{2}} \text {. }
$$

Then by unfolding one recovers $M_{1}$ and $M_{2}$.

Coming back to the spin-c structure on $M$ which we described above, let $\not \partial$ be the spin-c Dirac operator. Since the index of this operator only depends on $\omega$, we will denote it by $\operatorname{Ind}(M)$. The second main result of this paper is the following formula for this index:

$$
\operatorname{Ind}(M)=\sum(-1)^{\sigma_{i}} \int_{M_{i}} \exp \left(\omega_{i}\right) \operatorname{Todd}\left(M_{i}\right),
$$

where we orient the $M_{i}$ 's symplectically, $\sigma_{i}=0$ if the orientation induced on $M_{i}$ by $M$ agrees with its symplectic orientation and $\sigma_{i}=1$ if not. For example, for the connected sum (1.3), this formula reduces to

$$
\operatorname{Ind}(M)=\operatorname{Ind}\left(M_{1}\right)-\operatorname{Ind}\left(M_{2}\right) .
$$

We will prove the formula above in $\S 8$. For the connected sum (1.3), an equivariant version of this formula is closely related to the equivariant index theorem proved by Grossberg and Karshon in [6, §12].

AcKnowledgements: Cannas, Karshon and Tolman have recently proved in [2] an analogue for spin-c manifolds of the "quantization commutes with reduction" theorems of [3], [7], [13, 14] and [16, 17], and there is some overlap between their results and the results reported on here. The proof of (1.4) is partly modeled on Meinrenken's proof of a similar result in [14]. We are grateful to the referee who made several corrections and pertinent remarks, and to Allen Knutson who alerted us to a serious sign mistake on an earlier version of this paper. Finally, we are indebted to Michèle Vergne for her kindness to us during our stay at the École Normale in June of 1995 (during which the first draft of this paper was written).

\section{Folded Symplectic Structures}

Let $M$ be a $2 d$-dimensional manifold and $\omega \in \Omega^{2}(M)$ a closed two-form. Let $Z$ be the set of points where $\omega^{d}$ is zero. If $\omega^{d}$ intersects the zero section of $\wedge^{2 d} T^{*} M$ tranversally, $Z$ will be a codimension one submanifold of $M$. Let us assume that $\omega^{d}$ has this property. We denote by $\iota$ the inclusion map of $Z$ into $M$.

Definition. If the form $\left(\iota^{*} \omega\right)^{d-1} \in \Omega^{2 d-2}(Z)$ is non-vanishing, $\omega$ is said to be a folded symplectic form and $Z$ its folding hypersurface.

For a proof that this definition is equivalent to the definition in $\S 1$, see the comments at the end of this section or see [11, p.157].

It is clear that the property of being folded is an open property: if $\omega_{0}$ is folded and $\omega$ is a closed two-form which is $C^{1}$-close to $\omega_{0}$, then $\omega$ is also folded. However, 
this property is far from being generic: In dimension 4 it is generically true that $\omega^{2}$ is transverse to zero, but not that $\iota^{*} \omega$ is non-vanishing; in dimensions 6 and higher even the first assertion is false. (In particular, the set $Z$ is not a manifold generically except in dimension 4 . See [11] for a discussion of generic singularities of closed two-forms.)

Suppose now that $M$ is oriented. Then $Z$ acquires from $M$ a canonical orientation in the following manner: Let $M^{+}$be the set of points, $p$, where $\omega_{p}^{d}>0$ and $M^{-}$the set where $\omega_{p}^{d}<0$. Then

$$
M \backslash Z=M^{+} \cup M^{-},
$$

and by the tubular neighborhood theorem one gets from (2.1) an orientation of the normal bundle of $Z$ and hence of $Z$ itself.

Let $E \rightarrow Z$ be the rank 2 vector bundle whose fiber at $z$ is the annihilator of $\omega$ in $T_{z} M$. From the $(2 d-2)$-form $\omega^{d-1}$ one gets an orientation of the quotient bundle $\left(\iota^{*} T M\right) / E$, and hence, from the orientation of $T M$, an orientation of $E$. Moreover, from the orientations of $E$ and of $T Z$, one gets an orientation of the intersection of these two bundles, which is a rank-one subbundle, $F$, of $T Z$. Let $v$ be an oriented non-vanishing section of $F$ (i.e., a vector field with the property that $v_{z} \in F_{z}^{+}$for all $z \in Z$ ) and let $\alpha \in \Omega^{1}(Z)$ be a one-form for which $\imath_{v} \alpha=1$. We will need in $\S 8$ the following global variant of (1.1).

Theorem 1. Suppose that $Z$ is compact. Then there exists a neighborhood, $\mathcal{U}$, of $Z$ and an orientation preserving diffeomorphism,

$$
\varphi: Z \times(-\varepsilon, \varepsilon) \longrightarrow \mathcal{U},
$$

such that

$$
\varphi \circ \iota_{0}=\iota
$$

and

$$
\varphi^{*} \omega=\pi^{*} \iota^{*} \omega+d\left(t^{2} \pi^{*} \alpha\right),
$$

$\iota_{0}$ being the inclusion map $z \mapsto(z, 0), \pi$ the projection $(z, t) \mapsto z$, and $t$ the coordinate function on $(-\varepsilon, \varepsilon)$.

Proof. Let $w$ be a vector field on $M$ such that, for all $z \in Z,\left(w_{z}, v_{z}\right)$ is an oriented basis of $E_{z}$, and for the moment let the map $\varphi$ in (2.2) be the map which takes lines $(z, t),-\varepsilon<t<\varepsilon$ onto the integral curves of $w$. If we require that (2.3) hold, this $\varphi$ will be unique. (It will not be the $\varphi$ that we want, but will turn out to be a good first approximation to it.) Via $\varphi$ we can identify $\mathcal{U}$ with $Z \times(-\varepsilon, \varepsilon)$ and $w$ with the vector field $\frac{\partial}{\partial t}$. Moreover, we can extend $v$ to all of $\mathcal{U}$ via the inclusion $T_{z} Z \hookrightarrow T_{(z, t)} \mathcal{U}$. Let $\pi$ as above be the projection of $\mathcal{U}$ onto $Z$ which maps $(z, t)$ to $z$. We will prove the theorem by applying the "Moser trick" to the forms

$$
\omega_{0}:=\pi^{*} \iota^{*} \omega+d\left(t^{2} \pi^{*} \alpha\right),
$$


and

$$
\omega_{1}:=\omega .
$$

For this we will need the following "folding" criterion:

Lemma 2.7. Let $\mu$ be a closed two-form on $\mathcal{U}$. Then the form $\pi^{*} \iota^{*} \omega+t \mu$ is a folded symplectic form on a possibly smaller open neighborhood, $\mathcal{U}_{1}=Z \times(-\varepsilon, \varepsilon)$, of $Z$ if and only if $\mu(w, v)$ is nonvanishing on $Z$.

Proof of Lemma 2.7. This follows from the fact that the $d$-th exterior power of this form is

$$
(d-1) t\left(\pi^{*} \iota^{*} \omega\right)^{d-1} \wedge \mu+O\left(t^{2}\right),
$$

so this form is folded if and only if $\left(\pi^{*} \iota^{*} \omega\right)^{d-1} \wedge \mu$ is nonvanishing on $Z$. However, the annihilator of $\pi^{*} \iota^{*} \omega_{z}$ in $T_{z} \mathcal{U}$ is spanned by $w_{z}$ and $v_{z}$; so the nonvanishing of this form is equivalent to the nonvanishing of $\mu(w, v)$.

It is clear from (2.5) that $\omega_{0}$ is of the form above, i.e.,

$$
\omega_{0}=\pi^{*} \iota^{*} \omega+t \mu_{0},
$$

where

$$
\mu_{0}:=2 d t \pi^{*} \alpha+t d\left(\pi^{*} \alpha\right)
$$

and the same is also true of $\omega$. To see this note that, since $w=\frac{\partial}{\partial t}, \imath_{w} \pi^{*} \iota^{*} \omega=0$. On the other hand, $\imath_{w} \omega=0$ on $Z$, so $\omega-\pi^{*} \iota^{*} \omega$ is zero at all points of $Z$. Since $Z$ is defined by the equation $t=0$, we conclude that $\omega-\pi^{*} \iota^{*} \omega=t \mu_{1}$, for $\mu_{1} \in \Omega^{2}(\mathcal{U})$.

By $(2.8), \mu_{0}(w, v)=2$ on $Z$; so, by Lemma $2.7, \omega_{0}$ is folded. Moreover, since $\omega$ is folded, $\mu_{1}(w, v)$ is nonvanishing on $Z$. In fact, because of the orientation conventions discussed above, $\mu_{1}(w, v)$ is positive on $Z$.

We will next show that $\omega_{0}$ can be deformed into $\omega_{1}$ by a "folded" homotopy:

Lemma 2.9. For $0 \leq s \leq 1$, the form

$$
\omega_{s}:=(1-s) \omega_{0}+s \omega_{1},
$$

is folded (with folding hypersurface $Z$ ).

Proof of Lemma 2.9. $\omega_{s}$ is of the form $\omega_{s}=\pi^{*} \iota^{*} \omega+t \mu_{s}$ where $\mu_{s}=(1-s) \mu_{0}+$ $s \mu_{1}$. Hence, the function $\mu_{s}(w, v)$ is positive on $Z$, and so, by Lemma $2.7, \omega_{s}$ is folded.

Therefore, $\imath_{v} \omega_{s}$ and $\imath_{w} \omega_{s}$ vanish on $Z$, and the following is an easy corollary of Lemma 2.9 .

Lemma 2.10. Given $\nu \in \Omega^{1}(\mathcal{U})$, one can find a vector field, $u$, with the property

$$
\imath_{u} \omega_{s}=\nu
$$

if and only if $\imath_{v} \nu$ and $\imath_{w} \nu$ vanish on $Z$. 
Remark. Since $\omega_{s}$ is symplectic on the complement of $Z$, this vector field, if it exists, is unique.

To prove Theorem 1, recall that by the "Moser trick" the proof can be reduced to finding a vector field, $v_{s}$, on $\mathcal{U}$ which vanishes on $Z$, depends smoothly on $s$, and satisfies

$$
D_{v_{s}} \omega_{s}=-\dot{\omega}_{s}=\omega_{0}-\omega_{1} .
$$

Indeed, if such a vector field exists, one can integrate the equation

$$
\frac{d \varphi_{s}}{d s} \circ \varphi_{s}^{-1}=v_{s}
$$

with $\varphi_{0}$ equal to the identity and $\varphi_{s} \circ \iota_{0}=\iota_{0}$, to get a diffeomorphism, $\varphi_{s}$, satisfying $\varphi_{s}^{*} \omega_{s}=\omega_{0}$. To solve $(2.11)$, one observes that $\omega_{0}-\omega_{1}$ is closed and vanishes on $Z$. Therefore, since $Z$ is a deformation retract of $\mathcal{U}$, there exists a one-form, $\nu$, which satisfies $d \nu=\omega_{0}-\omega_{1}$ and vanishes to the second order on $Z$. Hence, $(2.11)$ is equivalent to

$$
d \imath_{v_{s}} \omega_{s}=d \nu
$$

so it suffices to solve

$$
\imath_{v_{s}} \omega_{s}=\nu
$$

and, by Lemma 2.10, there exists a unique $v_{s}$ satisfying (2.12) (and $v_{s}$ has to vanish to first order on $Z$.)

\section{Remarks.}

1. From Theorem 1 and the classical Darboux theorem, one gets another proof of (1.1). (By the classical Darboux theorem, $i^{*} \omega=d x_{2} d y_{2}+\ldots+d x_{d} d y_{d}$. Now apply Theorem 1 with $x_{1}=t$ and $\alpha=d y_{1}$.)

2. Let $G$ be a compact Lie group. If $G$ acts on $M$ and this action preserves $\omega$, one can arrange for $\alpha$ to be $G$-invariant and the map (2.2) to be $G$ equivariant.

3. If $Z$ is not compact, the assertion of Theorem 1 is still true provided we replace $\varepsilon$ by an appropriate continuous function $\varepsilon: Z \rightarrow \mathbb{R}^{+}$.

\section{Examples}

1. If we regard the even-dimensional sphere $S^{2 d}$ as the set of unit vectors in $\mathbb{R}^{2 d+1}$, we obtain a folded symplectic form on $S^{2 d}$ by restricting to $S^{2 d}$ the form

$$
d x_{1} \wedge d y_{1}+\ldots+d x_{d} \wedge d y_{d} .
$$

The folding hypersurface is the equator $S^{2 d} \cap\left\{x_{d+1}=0\right\}$.

Alternatively, this folded symplectic form on $S^{2 d}$ may be obtained by doubling a $2 d$-dimensional disk equipped with the standard symplectic form, $d x_{1} \wedge d y_{1}+$ $\ldots+d x_{d} \wedge d y_{d}$, i.e., gluing two $2 d$-dimensional disks equipped with standard symplectic forms along their sphere boundaries, after reversing the orientation on one of the disks. 
Yet a third way to construct this folded symplectic structure on $S^{2 d}$ justifies the name "folded". Consider the folding map from the sphere to the disk,

$$
\pi: S^{2 d} \longrightarrow D^{2 d},
$$

folding along the equator. Let $\nu=d x_{1} \wedge d y_{1}+\ldots+d x_{d} \wedge d y_{d}$ be the standard symplectic form on $D^{2 d}$. Then $\omega=\pi^{*} \nu$ is a folded symplectic form on $S^{2 d}$.

2. Let $\left(M_{1}, \omega_{1}\right)$ and $\left(M_{2}, \omega_{2}\right)$ be compact symplectic manifolds of dimension $2 d$ and with orientations induced by the symplectic forms. Let

$$
M=M_{1} \# \overline{M_{2}}
$$

be the connected sum, where $\overline{M_{2}}$ has the opposite of the symplectic orientation. Then $M$ has a folded symplectic form which coincides with the $\omega_{i}$ 's away from a tubular neighborhood of the surgery. To see this, concentrate on the small annuli $A_{i} \simeq S^{2 d-1} \times I, i=1,2$, where the surgery occurs. The symplectic form $\omega_{i}$ restricted to $A_{i}$ is diffeomorphic to $d\left(r_{i} \wedge \pi^{*} \alpha\right), i=1,2$, where $r_{i}$ is a coordinate on $I, \pi$ is the projection $S^{2 d-1} \times I \rightarrow S^{2 d-1}$ and $\alpha$ is the standard contact one-form on $S^{2 d-1}$. Choose coordinates $t_{1}, t_{2}$ such that $r_{i}=1+t_{i}^{2}$ for $t_{i}>\varepsilon$. Finally, extend $\omega$ across the connected sum by defining it to be

$$
\omega=d\left[\left(1+t^{2}\right) \wedge \pi^{*} \alpha\right],
$$

where $t=-t_{1}$ on the interval $t<-\varepsilon$ and $t=t_{2}$ on the interval $t>\varepsilon$. The folding hypersurface of $\omega$ is given by $t=0$. For a generalization of this folding construction, see $\S 6$.

3. The product of any contact manifold with the circle admits a folded symplectic form. This can be seen by doubling a symplectization of the contact manifold.

In particular, since Martinet [10] proved that any orientable 3-manifold admits a contact form, we conclude that the product of any orientable 3-manifold with the circle admits a folded symplectic form.

More generally, given a contactomorphism $\psi$ of a contact manifold $X$, consider the mapping torus $M_{\psi}=X \times[0,1] / \sim$, where $(p, 0) \sim(\psi(p), 1)$. The symplectization $X \times[0,1]$ has a $\omega$-concave boundary, say $X \times\{0\}$, and a $\omega$-convex boundary, say $X \times\{1\}$. There is a folded symplectic form on $M_{\psi}$ obtained by gluing $X \times[0,1]$ to $\overline{X \times[0,1]}$ under the identification by $\psi$ of the $\omega$-convex boundaries and the identity identification of the $\omega$-concave boundaries.

4. The notion of folded symplectic form holds for arbitrary even-dimensional manifolds, not necessarily orientable. For instance, the folded symplectic form on $S^{2 d}$ which we first described is invariant under the involution $x \mapsto-x$, and hence induces a folded symplectic form on the real even-dimensional projective spaces, $\mathbb{R} \mathbb{P}^{2 d}$.

It follows that the real blow-up of a folded symplectic manifold at a point (away from the folding hypersurface) admits a folded symplectic form. In fact, 
the real blow-up at a point of a $2 d$-dimensional manifold $M$ amounts to taking the connected sum of $M$ with $\mathbb{R P}^{2 d}$.

\section{Stable Complex Structures}

Let $\mathcal{U}$ be a neighborhood of the folding hypersurface in $M$ as in Theorem 1. Since $\omega$ is symplectic on $M \backslash \mathcal{U}$, there exists an almost complex structure, $J_{0}$, on $M \backslash \mathcal{U}$ which is compatible with $\omega$, in the sense that, for all $p \in M \backslash \mathcal{U}$, the map

$$
r, s \in T_{p} M \longmapsto \omega_{p}(J r, s),
$$

is a positive definite symmetric bilinear form. It is clear from the orientation considerations that $J_{0}$ cannot be extended to all of $M$; however, we will prove that one can add a trivial $\mathbb{R}^{2}$ bundle to $T M$ and extend the complex structure

$$
\begin{cases}J_{0} \oplus\left(\text { rotation by } 90^{\circ}\right) & \text { on } M^{+} \backslash \mathcal{U} \\ J_{0} \oplus\left(\text { rotation by }-90^{\circ}\right) & \text { on } M^{-} \backslash \mathcal{U} .\end{cases}
$$

to all of $M$ :

Theorem 2. There exists a complex structure, $J$, on the real $(2 d+2)$-dimensional vector bundle $T M \oplus \mathbb{R}^{2}$, and a $\mathbb{C}$-linear isomorphism

$$
\left(T M \oplus \mathbb{R}^{2}\right)_{M \backslash \mathcal{U}} \simeq T(M \backslash \mathcal{U}) \oplus \mathbb{C} .
$$

Moreover, $T M \oplus \mathbb{R}^{2}$ has a structure of symplectic vector bundle which is canonical up to homotopy, and the homotopy class of $J$ is unique provided $J$ is compatible with the symplectic structure on $T M \oplus \mathbb{R}^{2}$.

Proof. Let $E$ be the vector subbundle of $T \mathcal{U}$ spanned by the vector fields $w$ and $v$, as in $\S 2$. This is a symplectic subbundle of $T \mathcal{U}$, and we will denote by $E^{\perp}$ its symplectic orthocomplement. Without loss of generality, one can assume that $J_{0}$ extends over the set $|t| \geq \frac{\varepsilon}{2}$, and, on this set, is the sum of complex structures on $E$ and $E^{\perp}$. One can also assume that the complex structure on $E^{\perp}$ extends over all of $\mathcal{U}$ and that on the set $t \leq-\frac{\varepsilon}{2}$ the complex structure on $E$ is given by

$$
J_{0} w=-v \quad \text { and } \quad J_{0} v=w,
$$

and on the set $t \geq \frac{\varepsilon}{2}$ by

$$
J_{0} w=v \quad \text { and } \quad J_{0} v=-w .
$$

Consider, for $0 \leq \theta \leq \pi$, the $4 \times 4$ matrix:

$$
A_{\theta}:=\left[\begin{array}{cccc}
0 & \cos \theta & 0 & \sin \theta \\
-\cos \theta & 0 & \sin \theta & 0 \\
0 & -\sin \theta & 0 & \cos \theta \\
-\sin \theta & 0 & -\cos \theta & 0
\end{array}\right] .
$$

It is easy to check that $A_{\theta}^{2}=-\mathrm{Id}, A_{\theta}^{t}=-A_{\theta}$ and $A_{0}=-A_{\pi}$. Let $B_{t}=A_{\theta}$ where $\theta=\frac{\pi}{\varepsilon} t+\frac{\pi}{2}$. By (4.2) and (4.3), $B_{t}$ defines a complex structure on the bundle $E \oplus \mathbb{R}^{2}$ over the set $|t| \leq \frac{\varepsilon}{2}$, which agrees with the complex structure (4.1) on $|t|=\frac{\varepsilon}{2}$ provided one identifies $\mathbb{R}^{2}$ with $\mathbb{C}$ over $t \leq-\frac{\varepsilon}{2}$ by the map $(x, y) \mapsto x+i y$, 
and over $t \geq \frac{\varepsilon}{2}$ by the map $(x, y) \mapsto x-i y$. (This structure can be made to depend smoothly on $t$ by modifying the parametrization slightly in the vicinity of $t= \pm \frac{\varepsilon}{2}$.) Since the complex structure on $E^{\perp}$ is already defined on all of $\mathcal{U}$, this concludes the proof of the first assertion of Theorem 2 .

Let $\Omega_{0}$ be the standard symplectic structure on $\mathbb{R}^{2}$. Over $M \backslash \mathcal{U}$, the canonical symplectic structure, $\Omega$, on $T M \oplus \mathbb{R}^{2}$ is

$$
\Omega= \begin{cases}\omega \oplus \Omega_{0} & \text { on } M^{+} \backslash \mathcal{U} \\ \omega \oplus\left(-\Omega_{0}\right) & \text { on } M^{-} \backslash \mathcal{U}\end{cases}
$$

On $\mathcal{U}$ consider the splitting (canonically unique up to homotopy)

$$
T \mathcal{U} \oplus \mathbb{R}^{2}=E^{\perp} \oplus E \oplus \mathbb{R}^{2} .
$$

On $E^{\perp}$, the form $\Omega$ is the restriction of $\omega$, and $E^{\perp}$ is $\Omega$-orthogonal to $E \oplus \mathbb{R}^{2}$. Let $(w, v, e, f)$ be an oriented orthonormal basis of $E \oplus \mathbb{R}^{2}$ with respect to a metric $\langle\cdot, \cdot\rangle$. We can assume that for $t \leq-\frac{\varepsilon}{2}$ the canonical symplectic structure on $E \oplus \mathbb{R}^{2}$ is given relative to this basis by

$$
\Omega(a, b)=\left\langle A_{0} a, b\right\rangle,
$$

and on the set $t \geq \frac{\varepsilon}{2}$ by

$$
\Omega(a, b)=\left\langle A_{\pi} a, b\right\rangle,
$$

where $A_{\theta}$ is as above. When $|t| \leq \frac{\varepsilon}{2}$, we declare the canonical symplectic structure on $E \oplus \mathbb{R}^{2}$ to be given by

$$
\Omega(a, b)=\left\langle B_{t} a, b\right\rangle,
$$

where $B_{t}=A_{\theta}$ for $\theta=\frac{\pi}{\varepsilon} t+\frac{\pi}{2}$. (In order to have smoothness in $t$ we need to modify the parametrization slightly in the vicinity of $t= \pm \frac{\varepsilon}{2}$.) This defines a canonical symplectic structure on the vector bundle $T M \oplus \mathbb{R}^{2}$, which is unique up to homotopy. ${ }^{2}$

The set of all $J$ 's compatible with a symplectic structure on $T M \oplus \mathbb{R}^{2}$ is contractible (see, for instance, [12, p.67]).

Remark. The $Z$-tangent bundle of $M,{ }^{Z} T M$, is the vector bundle over $M$ whose sections are the vector fields on $M$ which over $Z$ are tangent to $Z$. In coordinates $x_{1}, \ldots, x_{2 d}$ for an open subset $\mathcal{U}$ of $M$, if the equation $x_{1}=0$ describes $Z \cap \mathcal{U}$, then the vector fields

$$
x_{1} \frac{\partial}{\partial x_{1}}, \frac{\partial}{\partial x_{2}}, \ldots, \frac{\partial}{\partial x_{2 d}},
$$

span the space of sections of ${ }^{Z} T M$ over $\mathcal{U}$ as a $C^{\infty}(\mathcal{U})$-module. When $Z$ is separating, the $Z$-tangent bundle of $M$ is stably isomorphic to $T M$, where the

\footnotetext{
${ }^{2}$ Since $\pi_{1}(\mathrm{SO}(4))=\mathbb{Z}_{2}$, there are two non-homotopic paths connecting $A_{0}$ to $A_{\pi}$; the other choice is given by matrices analogous to the $A_{\theta}$ 's but with opposite signs along the anti-diagonal. We are declaring the canonical path $A_{\theta}$ to be as above.
} 
isomorphism is canonical up to homotopy. ${ }^{3}$ A folded symplectic form on $M$ induces a structure of complex vector bundle on ${ }^{Z} T M$ which is canonical up to homotopy.

A canonical stable complex structure on $T M$ induced by the folded symplectic form is homotopic to a canonical stable almost complex structure induced by the stable isomorphism ${ }^{Z} T M \simeq_{s} T M$.

\section{Spin-c Structures}

We will next describe how Theorem 2 can be used to define a spin-c structure on $M$. Recall that a spin-c structure is defined as follows. The spin group is the connected double covering

$$
\operatorname{Spin}(2 d) \stackrel{p}{\longrightarrow} \mathrm{SO}(2 d)
$$

with kernel $\mathbb{Z}_{2}=\{1,-1\}$. One takes the central extension of this:

$$
\operatorname{Spin}^{c}(2 d):=\operatorname{Spin}(2 d) \times_{\mathbb{Z}_{2}} \mathrm{U}(1),
$$

$\mathrm{U}(1)$ being the group of complex numbers of modulus one, and $\mathbb{Z}_{2}$ the subgroup generated by $(-1,-1)$. The map $(A, \lambda) \mapsto\left(p(A), \lambda^{2}\right)$ defines a double covering

$$
\operatorname{Spin}^{c}(2 d) \stackrel{\xi}{\longrightarrow} \mathrm{SO}(2 d) \times \mathrm{U}(1) .
$$

Suppose now that the manifold $M$ is oriented, and is equipped with a riemannian metric and a hermitian line bundle. From the orientation and the metric, one gets a principal $\mathrm{SO}(2 d)$ bundle, $P_{\mathrm{SO}(2 d)}$, namely the bundle of oriented orthonormal frames, and from the hermitian line bundle one gets an associated circle bundle, $P_{\mathrm{U}(1)}$.

Definition. A spin-c structure is a principal $\operatorname{Spin}^{c}(2 d)$-bundle, $P_{\operatorname{Spin}^{c}(2 d)} \rightarrow M$, and a double covering 4

$$
P_{\mathrm{Spin}^{c}(2 d)} \longrightarrow P_{\mathrm{SO}(2 d)} \times P_{\mathrm{U}(1)},
$$

which is equivariant with respect to (5.1).

Let us now see how the complex structure on $T M \oplus \mathbb{R}^{2}$ enables us to get such a double covering. ${ }^{5}$ By $[8$, appendix D], there are canonical morphisms of Lie

\footnotetext{
${ }^{3}$ The construction of the $Z$-tangent bundle makes sense for $Z$ not necessarily separating. In general, ${ }^{Z} T M \oplus \mathbb{R} \simeq T M \oplus^{Z} L$ where ${ }^{Z} L$ is the line bundle dual to $Z$ (whose sections are the real functions on $M$ which are zero over $Z$ ). Therefore, the total Stiefel-Whitney class of $Z_{T M}$ is $w(T M)\left(1+[Z]^{\vee}\right)$, where $w(T M)$ is the total Stiefel-Whitney class of $M$ and $[Z]^{\vee}$ is the reduction modulo 2 of the Poincaré dual of the homology class of $Z$.

${ }^{4}$ The product $\times$ is the fiber product here and elsewhere in the paragraphs below.

${ }^{5} \mathrm{~A}$ similar argument is contained in [2].
} 
groups

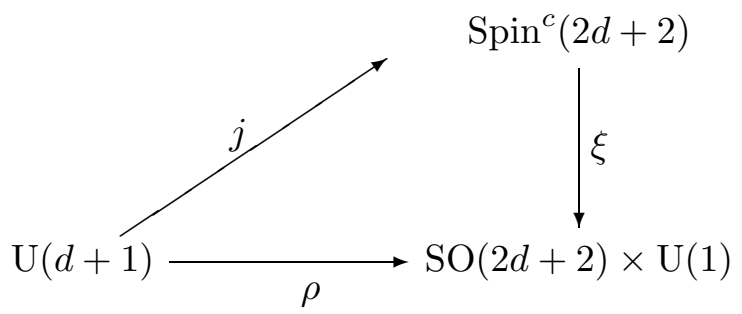

$\xi$ being the standard two-fold covering of $\mathrm{SO}(2 d+2) \times \mathrm{U}(1)$ by $\operatorname{Spin}^{c}(2 d+2), \rho$ the map "inclusion $\times \operatorname{det}$ " and $j$ its canonical lifting to $\operatorname{Spin}^{c}(2 d+2)$ (loc. cit., formula D.10).

Let $H$ be a hermitian inner product on $T M \oplus \mathbb{R}^{2}$ which is compatible with $J$ and let $P_{\mathrm{U}(d+1)}$ be the corresponding unitary frame bundle. Setting

$$
\begin{aligned}
P_{\mathrm{U}(1)} & :=P_{\mathrm{U}(d+1)} \times{ }_{\operatorname{det}} \mathrm{U}(1), \\
P_{\mathrm{SO}(2 d+2)} & :=P_{\mathrm{U}(d+1)} \times{ }_{\text {inclusion }} \mathrm{SO}(2 d+2), \quad \text { and } \\
P_{\mathrm{Spin}^{c}(2 d+2)} & :=P_{\mathrm{U}(d+1)} \times{ }_{j} \operatorname{Spin}^{c}(2 d+2),
\end{aligned}
$$

one gets from (5.2) morphisms of bundles

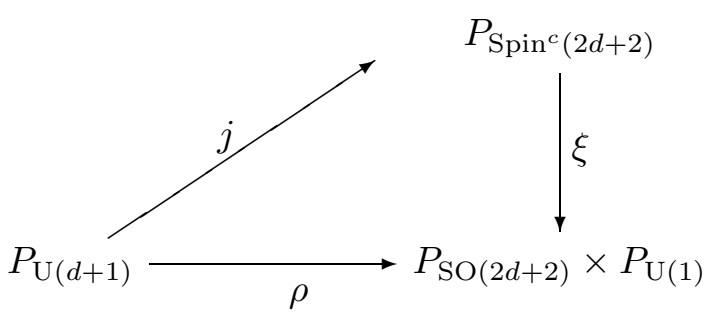

On the other hand, by restricting $\operatorname{Re} H$ to $T M$, one gets a riemannian metric on $M$ and a bundle of oriented orthonormal frames, $P_{\mathrm{SO}(2 d)}$. Moreover, there is a natural inclusion

$$
\eta: P_{\mathrm{SO}(2 d)} \times P_{\mathrm{U}(1)} \longrightarrow P_{\mathrm{SO}(2 d+2)} \times P_{\mathrm{U}(1)} .
$$

Let $P_{\mathrm{Spin}^{c}(2 d)}$ be the set of all pairs $(z, w), z$ in $P_{\mathrm{SO}(2 d)} \times P_{\mathrm{U}(1)}$ and $w$ in $P_{\text {Spin }^{c}(2 d+2)}$, such that $\eta(z)=\xi(w)$, and let $\kappa$ be the map

$$
P_{\mathrm{Spin}^{c}(2 d)} \longrightarrow P_{\mathrm{SO}(2 d)} \times P_{\mathrm{U}(1)},
$$

mapping $(z, w)$ to $z$. This map is a double covering. Furthermore, because of the commutativity of the maps

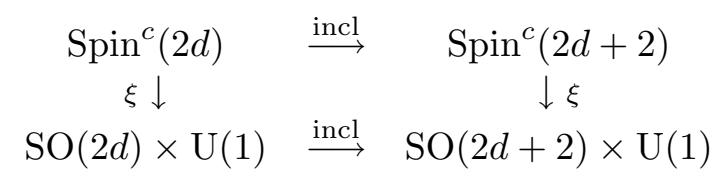

the action of $\operatorname{Spin}^{c}(2 d)$ on $P_{\operatorname{Spin}^{c}(2 d+2)}$ leaves $P_{\operatorname{Spin}^{c}(2 d)}$ fixed (as a set) and makes $P_{\text {Spinin }^{c}(2 d)}$ into a principal $\operatorname{Spin}^{c}(2 d)$-bundle. Thus (5.4) defines a spin-c structure 
on $M$. By (5.3), the line bundle associated with this spin-c structure, i.e., the line bundle corresponding to $P_{\mathrm{U}(1)}$, is

$$
\bigwedge_{\mathbb{C}}^{d+1}\left(T M \oplus \mathbb{R}^{2}\right) .
$$

From this spin-c structure one can get other spin-c structures by twisting with line bundles. The general principle is the following: Let $G$ be a Lie group which contains U(1) as a central subgroup, and let $P_{G} \rightarrow M$ be a principal $G$-bundle and $L_{\alpha} \rightarrow M$ an hermitian line bundle. Let $P_{\mathrm{U}(1)}^{\alpha}$ be the circle bundle associated with $L_{\alpha}$. On the fiber product

$$
P_{G} \times P_{\mathrm{U}(1)}^{\alpha},
$$

$G$ acts by its action on the left factor and $\mathrm{U}(1)$ acts by its diagonal action. The $\mathrm{U}(1)$-action commutes with the action of $G$, so the quotient

$$
Q_{G}^{\alpha}:=\left(P_{G} \times P_{\mathrm{U}(1)}^{\alpha}\right) / \mathrm{U}(1)
$$

has a residual $G$-action which makes it into a principal $G$-bundle. We will call this the twisting of $P_{G}$ by $L_{\alpha}$.

One can apply this construction to $P_{\operatorname{Spin}^{c}(2 d)}$, since $\operatorname{Spin}^{c}(2 d)$ contains U(1) as a central subgroup. Let $P_{\mathrm{U}(1)}^{2 \alpha}$ be the circle bundle corresponding to the line bundle $L_{\alpha}^{2}$, and let $Q_{\mathrm{U}(1)}^{2 \alpha}$ be the twisting of $P_{\mathrm{U}(1)}$ by $P_{\mathrm{U}(1)}^{2 \alpha}$. The double covering (5.4) is equivariant with respect to the homomorphism

$$
\gamma: \mathrm{U}(1) \longrightarrow \mathrm{U}(1), \quad \gamma(a)=a^{2} .
$$

The double covering

$$
P_{\mathrm{U}(1)}^{\alpha} \longrightarrow P_{\mathrm{U}(1)}^{2 \alpha}
$$

is also equivariant with respect to $\gamma$. By taking the quotient of each side of the four-fold covering

$$
P_{\mathrm{Spin}^{c}(2 d)} \times P_{\mathrm{U}(1)}^{\alpha} \longrightarrow\left(P_{\mathrm{SO}(2 d)} \times P_{\mathrm{U}(1)}\right) \times P_{\mathrm{U}(1)}^{2 \alpha}
$$

by the diagonal action of $\mathrm{U}(1)$ via $\gamma$, one gets a double covering

$$
Q_{\mathrm{Spin}^{c}(2 d)}^{\alpha} \longrightarrow P_{\mathrm{SO}(2 d)} \times Q_{\mathrm{U}(1)}^{2 \alpha}
$$

We will call this the twisting of the spin-c structure (5.4) by $L_{\alpha}$. Note that, by (5.5), the line bundle defined by $Q_{\mathrm{U}(1)}^{2 \alpha}$ is the tensor product

$$
\bigwedge_{\mathbb{C}}^{d+1}\left(T M \oplus \mathbb{R}^{2}\right) \otimes L_{\alpha}^{2} .
$$

In particular, if the folded symplectic form that we started with is integral, i.e., $[\omega]$ is the image in $H^{2}(M ; \mathbb{R})$ of a cohomology class $\alpha \in H^{2}(M ; \mathbb{Z})$ and $L_{\alpha}$ is the line bundle whose Chern class is $\alpha$, we will call (5.6) the canonical spin-c structure on our folded symplectic manifold $M$. Since this structure depends on 
$J, H$, etc., this definition is a bit of a misnomer. However, it is to some extent justified by the following result.

Theorem 3. If $\not \partial$ is the spin-c Dirac operator associated with this spin-c structure, the index of $\not \partial$ is given by the formula

$$
\int_{M} \exp (\omega) \operatorname{Todd}\left(T M \oplus \mathbb{R}^{2}, J\right) .
$$

Proof. This is just a special case of the Atiyah-Singer formula for the spin-c Dirac operator. (See, for instance, [8], formula D.20.)

Note that the Todd class of $T M \oplus \mathbb{R}^{2}$ sits in the rational cohomology of $M$, so it is unchanged by smooth isotopies of $J$. Therefore, since $J$ is determined up to isotopy by $\omega$, it only depends on $\omega$ and hence so does the expression (5.7). Thus the index of $\not \partial$ is a folded symplectic invariant of $M$.

\section{Folding}

Let $W=W^{2 d}$ be a compact manifold with boundary equipped with a symplectic form, $\omega$. Theorem 1, the normal form theorem for folded symplectic forms, has the following analogue for manifolds with boundary. Let $t: W \rightarrow \mathbb{R}^{+}$ be a defining function for the boundary, i.e.,

$$
p \in \partial W \Leftrightarrow t(p)=0 \quad \text { and } \quad d t_{p} \neq 0, \forall p \in \partial W,
$$

and let $v$ be the hamiltonian vector field associated with $t$. By (6.1), $v$ is tangent to the boundary and on the boundary is nonvanishing. Let $\alpha$ be a one-form on $\partial W$ with the property $\imath(v) \alpha=1$.

Theorem 4. There exists a collar neighborhood, $\mathcal{U}=\partial W \times[0, \varepsilon)$, of $\partial W$ on which $\omega$ is diffeomorphic to

$$
\pi^{*} \iota^{*} \omega+d\left(t \pi^{*} \alpha\right)
$$

$\iota$ being the inclusion map of $\partial W$ into $W$ and $\pi$ the projection $(p, t) \mapsto p$.

Proof. The boundary $\partial W$ is a coisotropic submanifold of $W$, and (6.2) has the same restriction to $\partial W$ as $\omega$, so this is a consequence of the coisotropic imbedding theorem of Gotay [5] and Weinstein [18].

Remark. Though $t$ and $v$ are not canonically defined, the orientation of the normal bundle of $\partial W$ defined by $d t$ is canonically defined and hence so is the orientation of the null-foliation of $\partial W$ defined by $v$.

We will now describe a folding result which one can obtain from this theorem. Let $W_{1}$ and $W_{2}$ be compact oriented $2 d$-dimensional manifolds with boundary. Suppose that $\sigma: \mathcal{U}_{1} \rightarrow \mathcal{U}_{2}$ is an orientation-reversing diffeomorphism of collar neighborhoods of the boundaries, $\mathcal{U}_{i} \simeq \partial W_{i} \times I, i=1,2$, inducing an orientationreversing diffeomorphism $\rho$ of the boundaries. Let $M$ be the compact oriented manifold (without boundary) that one gets from

$$
W_{1} \sqcup \overline{W_{2}}
$$


by identifying $\mathcal{U}_{1}$ with $\overline{\mathcal{U}_{2}}$ via $\sigma$, where $\overline{W_{2}}$ denotes the manifold $W_{2}$ equipped with the opposite orientation. Let $\omega_{i}$ be a symplectic form on $W_{i}$. We orient $W_{1}$ and $W_{2}$ by their symplectic orientations; the orientations in $W_{1} \sqcup \overline{W_{2}}$ patch together to define an orientation of $M$. Let $\iota_{i}$ be the inclusion of $Z$ into $W_{i}$ and suppose that

$$
\iota_{1}^{*} \omega_{1}=\rho^{*} \iota_{2}^{*} \omega_{2}=\mu
$$

Suppose also that the two orientations of the null-foliation agree. By Theorem 4 , we may assume that the collar neighborhoods are of the form $\mathcal{U}_{i} \simeq Z \times\left[0, \varepsilon_{i}\right)$, and that, on $\mathcal{U}_{i}, \omega_{i}$ is diffeomorphic to

$$
\pi^{*} \mu+d\left(t_{i} \pi^{*} \alpha\right), \quad i=1,2 .
$$

Define a folded symplectic form, $\omega$, on $M$ by setting $\omega=\omega_{i}$ on $W_{i} \backslash \mathcal{U}_{i}$ and setting

$$
\omega=\pi^{*} \mu+d\left(t^{2} \pi^{*} \alpha\right)
$$

on $Z \times\left(-\delta_{2}, \delta_{1}\right)$, where $\delta_{i}^{2}=\varepsilon_{i}$ and $t$ is a coordinate function on the interval $\left(-\delta_{2}, \delta_{1}\right)$, which satisfies

$$
t^{2}=t_{1} \quad \text { on } \quad \frac{\delta_{1}}{2}<t<\delta_{1},
$$

and

$$
t^{2}=t_{2} \quad \text { on } \quad-\delta_{2}<t<-\frac{\delta_{2}}{2} .
$$

Since $\mathcal{U}_{1}$ and $\mathcal{U}_{2}$ can be chosen to be arbitrarily small, we have proved:

Theorem 5. For every neighborhood, $\mathcal{U}$, of $Z$, there exists a folded symplectic form on $M$ with folding hypersurface $Z$ such that $\omega=\omega_{i}$ on $W_{i} \backslash \mathcal{U}$.

The construction we have just described will be called folding. We will next describe an analogous "unfolding" construction.

\section{Unfolding}

Let $(M, \omega)$ be a compact oriented folded symplectic manifold, and let $Z$ be its folding hypersurface. For the moment we will assume that $M$ and $Z$ are connected and hence that $M \backslash Z$ consists of two connected components. We will denote their closures by $W_{1}$ and $W_{2}$. These are manifolds-with-boundary with the common boundary $Z$. Let $\mathcal{U}=Z \times(-\varepsilon, \varepsilon)$ be a tubular neighborhood of $Z$ in $M$ on which $\omega$ has the normal form

$$
\pi^{*} \iota^{*} \omega+d\left(t^{2} \pi^{*} \alpha\right)
$$

and let $\mathcal{U}_{1}=Z \times[0, \varepsilon)$ and $\mathcal{U}_{2}=Z \times(-\varepsilon, 0]$ be the intersections of $\mathcal{U}$ with $W_{1}$ and $W_{2}$ respectively. Let $t_{1}$ be a coordinate function on the interval $\left[0, \varepsilon^{2}\right)$ such that $t_{1}=t^{2}$ on the interval $\frac{\varepsilon}{2}<t<\varepsilon$, and let $t_{2}$ be a coordinate function on the interval $\left[0, \varepsilon^{2}\right)$ such that $t_{2}=t^{2}$ on the interval $-\varepsilon<t<-\frac{\varepsilon}{2}$. Then the symplectic form

$$
\omega_{1}=\pi^{*} \iota^{*} \omega+d\left(t_{1} \pi^{*} \alpha\right)
$$


can be extended to a symplectic form on $W_{1}$ by setting it equal to $\omega$ on $W_{1} \backslash \mathcal{U}_{1}$ and the form

$$
\omega_{2}=\pi^{*} \iota^{*} \omega+d\left(t_{2} \pi^{*} \alpha\right)
$$

can be extended to a symplectic form on $W_{2}$ by setting it equal to $\omega$ on $W_{2} \backslash \mathcal{U}_{2}$. Thus, to summarize, we have proved:

Theorem 6. $M$ can be "unfolded" into two symplectic pieces, $\left(W_{i}, \omega_{i}\right), i=$ 1,2, which are compact manifolds-with-boundary, having $Z$ as their common boundary.

This result can be considerably improved if we assume that the null-foliation on $Z$ is fibrating, i.e., that there exists a fibration

$$
p: Z \rightarrow B
$$

whose base is a compact manifold, $B$, and whose fibers are the leaves of the null-foliation. Let $v$ be a nonvanishing vector field whose integral curves are the fibers, and let $f(b)$ be the period of the integral curve sitting over $b ; f(b)$ depends smoothly on $b$, so we can reparametrize these integral curves by replacing $v$ by the vector field

$$
w:=\frac{2 \pi}{f} v
$$

whose integral curves are of period $2 \pi$. This modified vector field generates an action of $S^{1}$ on $Z$, and makes the fibration (7.3) into a principal $S^{1}$-bundle. Let $\alpha$ be a connection form on this bundle, i.e., an $S^{1}$-invariant one-form satisfying $\imath(w) \alpha=1$. One can extend the action of $S^{1}$ to the neighborhood $\mathcal{U}_{1}=Z \times\left[0, \varepsilon_{1}\right)$ of $Z$ in $W_{1}$ by letting it act trivially on $\left[0, \varepsilon_{1}\right)$. This action preserves the form $\omega_{1}$; indeed, by $(7.1), \imath(w) \omega_{1}=-d t_{1}$, so this action is hamiltonian with moment map $t_{1}$. Now apply the "symplectic cutting" operation to $\mathcal{U}_{1}$. By symplectic reduction there is a unique symplectic form, $\omega_{B}$, on $B$ such that $p^{*} \omega_{B}=\iota_{1}^{*} \omega_{1}$. Let $\mathcal{U}_{1}^{0}=\mathcal{U}_{1} \backslash Z$. Symplectic cutting (cf. [9]) says that the disjoint union

$$
B \cup \mathcal{U}_{1}^{0}
$$

can be made into a symplectic manifold in such a way that $B$ imbeds into this manifold as a symplectic submanifold of codimension two. Moreover, one can do this without changing the symplectic form, $\omega_{1}$, on $\mathcal{U}_{1}^{0}$ except on a small open set

$$
0<t_{1}<\varepsilon_{1}^{\prime} \ll \varepsilon_{1} .
$$

This glues together with the symplectic form, $\omega_{1}$, on $W_{1} \backslash \mathcal{U}_{1}$ to give a symplectic structure to the disjoint union

$$
M_{1}=B \cup\left(W_{1} \backslash Z\right) .
$$

One can apply the same construction to $W_{2}$ and thus finally show: 
Theorem 7. If the null-foliation on $Z$ is fibrating, then $M$ can be "unfolded" into compact symplectic manifolds, $M_{1}$ and $M_{2}$, each of which contains $B$ as an imbedded codimension two hypersurface.

If the null-foliation is not fibrating, one can still obtain a result of this sort provided that each leaf of the null-foliation has an open neighborhood in $Z$ diffeomorphic to $S^{1} \times_{\mathbb{Z}_{n}} \mathbb{R}^{2 d-2}$, where the null-foliation sits as the directions tangent to $S^{1}$, and $\mathbb{Z}_{n}$ acts on $\mathbb{R}^{2 d-2}$ by rotations (i.e., provided that the nullfoliation is a "Seifert fibration"). Hence, there is a locally free action of $S^{1}$ on $Z$ whose orbits are the leaves of the null-foliation and the argument above suffices to show:

Theorem 8. If each leaf of the null-foliation on $Z$ has an open neighborhood in $Z$ diffeomorphic to $S^{1} \times_{\mathbb{Z}_{n}} \mathbb{R}^{2 d-2}$, where the null-foliation sits as the directions tangent to $S^{1}$, and $\mathbb{Z}_{n}$ acts on $\mathbb{R}^{2 d-2}$ by rotations, then $M$ can be "unfolded" into compact symplectic orbifolds, $M_{1}$ and $M_{2}$, each of which contains $B$ as an imbedded codimension two symplectic suborbifold.

We have also been assuming up to this point that $Z$ is connected. This hypothesis can be relaxed. If the connected components of $M \backslash Z$ are $W_{i}^{0}$, $i=1, \ldots, N$, one can unfold $Z$, one component at a time, thus obtaining, in place of the $W_{i}^{0}$ 's, compact orbifolds, $M_{i}, i=1, \ldots, N$, just as above.

\section{Spin-c Index}

Let $M$ be a compact connected oriented $2 d$-dimensional manifold, and let $\omega \in \Omega^{2}(M)$ be a folded symplectic form with folding hypersurface $Z$. For simplicity we will assume that $Z$ is connected and hence that $M \backslash Z$ consists of two connected pieces. Suppose, as in $\S 7$, that the null-foliation on $Z$ is fibrating. Then, by Theorem $7, M$ unfolds into two compact symplectic manifolds, $\left(M_{i}, \omega_{i}\right), i=1,2$. We orient the $M_{i}$ 's by their symplectic orientations. Without loss of generality, we can assume that the (symplectic) orientation on $M_{1}$ coincides with the orientation induced by $M$, and that the (symplectic) orientation on $M_{2}$ is opposite to the orientation induced by $M$. The goal of this section is to compute the integral (5.7). (If $[\omega]$ is an integer cohomology class, this integral is, by Theorem 3, the index of the spin-c Dirac operator.) Explicitly we will prove:

Theorem 9. The integral (5.7) is equal to

$$
\int_{M_{1}} \exp \left(\omega_{1}\right) \operatorname{Todd}\left(M_{1}\right)-\int_{M_{2}} \exp \left(\omega_{2}\right) \operatorname{Todd}\left(M_{2}\right) .
$$

\section{Remarks.}

1. The cohomology classes $\left[\omega_{1}\right]$ and $\left[\omega_{2}\right]$ need not be integer cohomology classes. When they are, the two summands of (8.1) are just the RiemannRoch numbers of $M_{1}$ and $M_{2}$. 
2. If the null-foliation on $Z$ is not fibrating, but is as in Theorem 8 , then $M$ has an unfolding of the type in $\S 7$ where the $M_{i}$ 's are orbifolds. In this case, the formula (8.1) is still valid provided the terms on the right are replaced by the Kawasaki-Riemann-Roch numbers of $M_{1}$ and $M_{2}$.

3. If $Z$ is not connected, formula (8.1) has to be replaced by the slightly more complicated formula (1.4).

Proof. Choose a neighborhood, $\mathcal{U} \simeq Z \times(-\varepsilon, \varepsilon)$, of the folding hypersurface of the form given by Theorem 1, where, in the notation of $\S 2, \omega$ is diffeomorphic to

$$
\pi^{*} \iota^{*} \omega+d\left(t^{2} \pi^{*} \alpha\right) \text {. }
$$

The integral (5.7) can be written as a sum

$$
\begin{aligned}
\int_{M \backslash \mathcal{U}} \exp (\omega) & \operatorname{Todd}\left(T M \oplus \mathbb{R}^{2}, J\right) \\
& +\int_{\mathcal{U}} \exp (\omega) \operatorname{Todd}\left(T M \oplus \mathbb{R}^{2}, J\right) .
\end{aligned}
$$

Apply the "Meinrenken trick" to the integral (8.3) (cf. [14]): Pick a connection form, $\theta$, for the action of $S^{1}$ on $\mathcal{U}$, and note that, by Stokes' theorem, the integral (8.3) can be written as the value at $x=0$ of the function of $x$ given by

$$
\int_{\partial \mathcal{U}} \frac{\theta \exp (\omega) \operatorname{Todd}\left(T M \oplus \mathbb{R}^{2}, J\right)}{d \theta-x} .
$$

Now each of the two boundary components of $\partial \mathcal{U}$ can be identified with $Z$. Let $p: Z \rightarrow B$ be the fibration with total space $Z$ and symplectic base $\left(B, \omega_{B}\right)$, as in $\S 7$. The restriction of $T M \oplus \mathbb{R}^{2}$ to each of these boundary components is a complex vector bundle of the form

$$
p^{*} T B \oplus \mathbb{C}^{2},
$$

the complex structure on $T B$ being any complex structure which is compatible with $\omega_{B}$.

Thus the contribution of each of the boundary components is, up to sign (see below), equal to

$$
\int_{B} \frac{\exp \left(\omega_{B}\right) \operatorname{Todd}(T B)}{\mu-x}
$$

where $p^{*} \mu=d \theta$ (so $\mu$ is the curvature of the connection $\theta$ ).

However, the signs of these two contributions differ. The reason for this is that the orientation on $M$ induces on $(Z \times\{\varepsilon\}) / S^{1}$ an orientation which is compatible with $\omega_{B}^{d-1}$ and on $(Z \times\{-\varepsilon\}) / S^{1}$ an orientation which is compatible with $-\omega_{B}^{d-1}$.

Hence, the integral (8.3) is zero.

Consider now the expression (8.1). Regard $M_{i}$ as a union $\left(M_{i} \backslash \mathcal{U}_{i}\right) \cup \mathcal{U}_{i}$, where

$$
\mathcal{U}_{1}=B \cup(Z \times(0, \varepsilon))
$$


and

$$
\mathcal{U}_{2}=B \cup(Z \times(-\varepsilon, 0)) .
$$

Recall from $\S 7$ that the symplectic structures on $\mathcal{U}_{1}$ and $\mathcal{U}_{2}$ are acquired by applying the "symplectic cutting" operation to $Z \times(0, \varepsilon)$ and $Z \times(-\varepsilon, 0)$.

By assumption, $T M \oplus \mathbb{R}^{2}$ is isomorphic on $M \backslash \mathcal{U}$ to $T(M \backslash \mathcal{U}) \oplus \mathbb{C}$. Thus the summand (8.2) is equal to

$$
\int_{M_{1} \backslash \mathcal{U}_{1}} \exp \left(\omega_{1}\right) \operatorname{Todd}\left(M_{1}\right)-\int_{M_{2} \backslash \mathcal{U}_{2}} \exp \left(\omega_{2}\right) \operatorname{Todd}\left(M_{2}\right),
$$

where the minus sign follows from the mismatch between the (symplectic) orientation of $M_{2}$ and the orientation induced by $M$.

In order to prove the theorem it suffices to show that

$$
\int_{\mathcal{U}_{1}} \exp \left(\omega_{1}\right) \operatorname{Todd}\left(M_{1}\right)-\int_{\mathcal{U}_{2}} \exp \left(\omega_{2}\right) \operatorname{Todd}\left(M_{2}\right)
$$

is zero. To see this, note that, in view of the canonical form of Theorem 1 , one gets from the involution $t \mapsto-t$ a symplectomorphism from $M^{+} \cap \mathcal{U}$ onto $M^{-} \cap \mathcal{U}$, which descends to a symplectomorphism from $\mathcal{U}_{1}$ onto $\mathcal{U}_{2}$.

Remark. Let $G$ be a compact Lie group. If $G$ acts on $M$ and this action preserves $\omega$, then, by averaging, we can arrange that all auxiliary data is $G$-invariant, so that the index of the spin-c Dirac operator becomes a virtual representation of $G$. In this case, formula (8.1) holds as an isomorphism of virtual representations, yielding an equivariant version of Theorem 9 .

\section{References}

[1] M. Audin and J. Lafontaine, Eds., Holomorphic curves in symplectic geometry, Progress in Mathematics, 117, Birkhäuser Verlag, Basel, 1994.

[2] A. Cannas da Silva, Y. Karshon, and S. Tolman, Quantization of presymplectic manifolds and circle actions, to appear in Trans. Amer. Math. Soc.

[3] J.J. Duistermaat, V. Guillemin, E. Meinrenken, and S. Wu, Symplectic reduction and Riemann-Roch for circle actions, Math. Res. Lett. 2 (1995), 259-266.

[4] Y. Eliashberg, On singularities of folding type, (English translation by E. Primrose of the Russian original), Izv. Akad. Nauk SSSR 34 (1970), 1119-1134.

[5] M. Gotay, On coisotropic imbeddings of presymplectic manifolds, Proc. Amer. Math. Soc. 84 (1982), 111-114.

[6] M. Grossberg and Y. Karshon, Equivariant index and the moment map for completely integrable torus actions, Adv. Math. 133 (1998), 185-223.

[7] L. Jeffrey and F. Kirwan, On localization and Riemann-Roch numbers for symplectic quotients, Quart. J. Math. Oxford Ser. (2) 47 (1996), 165-185.

[8] H. Lawson, M.-L. Michelsohn, Spin geometry, Princeton Mathematical Series, 38, Princeton University Press, Princeton, NJ, 1989.

[9] E. Lerman, Symplectic cuts, Math. Res. Letters 2 (1995), 247-258.

[10] J. Martinet, Formes de contact sur les variétés de dimension 3, Proceedings of Liverpool Singularities Symposium, II (1969/1970), pp. 142-163, Lecture Notes in Math., 209, Springer, Berlin, 1971.

[11] _ Sur les singularités des formes différentielles, Ann. Inst. Fourier (Grenoble) 20 (1970), 95-178. 
[12] D. McDuff and D. Salamon, Introduction to symplectic topology, Oxford University Press, New York, 1995.

[13] E. Meinrenken, On Riemann-Roch formulas for multiplicities, J. Amer. Math. Soc. 9 (1996), 373-389.

[14] , Symplectic surgery and the spin ${ }^{c}$-Dirac operator, Adv. in Math. 134 (1998), 240-277.

[15] R. Melrose, Equivalence of glancing hypersurfaces, II, Math. Ann. 255 (1981), 159-198.

[16] M. Vergne, Geometric quantization and equivariant cohomology, First European Congress in Mathematics, Vol. I (Paris, 1992), pp. 249-295, Progr. Math., 119, Birkhäuser, Boston, 1994.

[17] _ Multiplicity formula for geometric quantization, I, II, Duke Math. J. 82 (1996), 143-179 and 181-194.

[18] A. Weinstein, Lectures on symplectic manifolds, CBMS Regional Conference Series in Mathematics, 29, American Mathematical Society, Providence, R.I., 1979.

Department of Mathematics, University of California, Berkeley, CA 94720.

E-mail address: acannas@math.berkeley.edu

Department of Mathematics, Massachusetts Institute of Technology, Cambridge, MA 02139.

E-mail address: vwg@math.mit.edu

Department of Mathematics, Rutgers University, Piscataway, NJ 08854.

E-mail address: ctw@math.rutgers.edu 